2 Hillman BJ. Evaluating magnetic resonance: what do we need to know? Invest Radiol 1986;21:289-9

3 St Leger AS, Allen D, Rowsell KV. Procedures for evaluating innovatory proposals. $B M f$ 1989;299:1017-8

4 Dixon AK, Kelsey FI, Kingham JGC, McLean AM, White FE. Computed tomography in patients with an abdominal mass: effective and efficient? A controlled trial. Lancet 1981;i:1199-201.

5 Russell I, Williams A. Evaluation of computerised tomography: a review of research methods. In: Culyer AJ, Horisberger B, eds. Economic and medical evaluation of health care techniques. Berlin: Springer Verlag, 1983:298-379.

6 Anonvmous. Body computed tomography: wanted or needed? [Editorial]. Lancet 1984;ii:961-2.

Morre AT, Dixon AK, Rubenstein D, Wheeler T. Cost benefit evaluation of body computed tomography. Health Trends 1987;19:8-12.

8 Cooper LS, Chalmers TC, McCally M, Berrier J, Sacks HS. The poor quality of early evaluations of magnetic resonance imaging. fAMA 1988;259: 3277-80.

9 Shuman WP. The poor quality of early evaluations of MR imaging: a reply A7R 1988:151:857-8.

10 Hillman $B J$. The assessment of $M R$ imaging. $A 7 R$ 1988:151:858-60.
11 Siegel S, Castellau NJ. Nonparametric statistics for the behavioural sciences. New Fork: McGraw-Hill, 1988:298-303.

12 Gudex C, Kind P. The QALY toolkit. York: Centre for Health Economics, University of York, 1988. (Discussion paper No 38 .

13 Kind P, Rosser R, Williams A. Valuation of quality of life: some psychometric evidence. In: Jones-Lee MW, ed. The value of life and safety. Amsterdam North-Holland, 1982:159-70.

14 Anonymous. The value of diagnostic tests [Editorial]. Lancet 1979;i:809-10.

5 Teasdale GM, Hadley DM, Lawrence A, et al. Comparison of magnetic resonance imaging and computed tomography in suspected lesions in the posterior cranial fossa. BMF 1989;299:349-55.

16 Franken EA, Berbaum KS, Dunn V, et al. Impact of MR imaging on clinical diagnosis and management: a prospective study. Radiology 1986;161: $377-80$.

17 Kent DL, Larson EB. Magnetic resonance imaging of the brain and spine-is clinical efficacy established after the first decade? Ann Intern Med 1988;108: $402-24$

18 Greenfield $S$. The state of outcome research: are we on target? $N$ Engl 7 . Med 1989;320:1142-3.

(Accepted 1 November 1990)
Stobhill Hospital, Glasgow J R Smith, MRCOG, research fellow, department of gynaecology

J Murdoch, MD, senior registrar in gynaecology A J Dougall, MRCPATH consultant bacteriologist C A Forrest, MRCOG, consultant gynaecologist J A Davis, FRCOG, consultant gynaecologist

\section{Maryhill Health Centre,}

H MacKinnon, FRCGP, general practitioner

D Baillie, MRCGP, general practitioner

D M Byford, MRCGP, general practitioner

Royal Infirmary, Glasgow

D Carrington, MRCPATH,

consultant virologist

C E Frew, BSC, senior

virologist

Correspondence to:

Mr J R Smith, The Jefferis

Wing, St Mary's Hospital,

London W2.

BMF 1991;302:82-4

\title{
Prevalence of Chlamydia trachomatis infection in women having cervical smear tests
}

\author{
J R Smith, J Murdoch, D Carrington, C E Frew, A J Dougall, H MacKinnon, D Baillie, D M Byford, \\ C A Forrest, J A Davis
}

\section{Abstract}

Objective-To determine the prevalence of sexually transmitted diseases in patients with normal and abnormal cervical smears.

Design-A prospective study of asymptomatic women with normal cervical smears attending their general practitioner and newly referred patients with abnormal smears attending a colposcopy clinic.

Setting-A hospital based colposcopy clinic and an urban general practice (list size 5500) in north west Glasgow.

Subjects -197 asymptomatic women attending their general practitioner for cervical smear tests and 101 randomly selected patients attending the colposcopy clinic for investigation of abnormal smears

Main outcome measures-Presence of various sexually transmitted infections as determined by culture and serological tests.

Results-Of the 101 women with cytological abnormalities, six had current chlamydial infection proved by culture and none had gonococcal infection; of the 197 women with normal smears, $24(12 \%)$ had a chlamydial infection and two had gonorrhoea. Serological studies for Chlamydia trachomatis specific antibody also indicated that a large proportion of patients had been exposed to this agent in both groups. There was no significant difference between the groups in the prevalence of any sexually transmitted disease studied.

Conclusion-A high prevalence of chlamydial infection is present in women in north west Glasgow irrespective of their cervical cytological state.

\section{Introduction}

The prevalence of Chlamydia trachomatis, Neisseria gonorrhoeae, herpes simplex virus, Candida albicans, and Trichomonas vaginalis infections in women varies with the clinical setting. In previous studies of asymptomatic women in general practice the prevalences of $C$ trachomatis and $N$ gonorrhoeae were $10.7 \%^{1}$ and $0.9 \%^{2}$ respectively, and prevalences of $3-8 \%^{34}$ and $1 \cdot 8 \%$ respectively were recorded in hospital gynaecology practice. Controversy surrounds the prevalence of chlamydial infection as highly variable rates have been measured in different studies. Screening tests for chlamydia have not been readily available to genera practitioners, and many doctors are not aware of the local prevalence rate. This is an important factor when considering whether to screen asymptomatic patients.

Cervical intraepithelial neoplasia is associated with exposure to multiple sexual contacts, a feature which also predisposes to the acquisition of sexually transmitted diseases. ${ }^{6}$ No study has compared the prevalence of sexually transmitted diseases in patients attending a colposcopy clinic for cytological abnormalities with that in patients in general practice from the same area whose smears are normal. We report such a study in north west Glasgow.

Patients and methods

REFERRALS TO COLPOSCOPY CLINIC

One hundred and one patients attending the colposcopy clinic at Stobhill Hospital were enrolled over one year. All were a subsample of consecutive new referrals selected at random. They had abnormal cytological findings as categorised by Papanicolaou class II with atypia (borderline nuclear abnormality; 20 cases) class III (mild to moderate dyskaryosis; 72 cases), class IV (severe dyskaryosis; eight cases), or class V (malignant cells; one case). All socioeconomic classes were represented, but most patients were in social class III. The age range of the patients was 19-58 years (mean 30)

At the initial visit full microbiological investigation was performed for $C$ trachomatis, $N$ gonorrhoeae, $T$ vaginalis, $C$ albicans, and herpes simplex virus. Swabs taken from the urethra, endocervix, and rectum were directly plated on to Thayer's medium for culture of $N$ gonorrhoeae and the plates incubated at $37^{\circ} \mathrm{C}$ in a carbon dioxide enriched atmosphere for 48 hours. Cervical smears were obtained using an Ayres spatula. Tests for $C$ trachomatis were performed by culture in McCoy cells on swab samples obtained from the endocervix. Positive cultures were identified when monoclonal antibodies (Microtrak-SYVA, United Kingdom) bound successfully to chlamydial inclusions.

In addition, blood was drawn for serological tests for chlamydia. Microimmunofluorescence tests were performed for $C$ trachomatis strains $\mathrm{D}-\mathrm{K}$ by using an antigen pool (IOL P2). Reciprocal antibody titres of $C$ trachomatis specific IgG and IgM were evaluated for each sample and interpreted as: IgG titre $<16$, probably no previous exposure to a chlamydial agent; IgG titre $>128$, suggestive evidence of recent infection; IgG 
titre 16-128, evidence of past infection; IgM positive, evidence of probable current infection. Similarly, tests for Chlamydia psittaci (IOL 395) and Chlamydia pneumoniae (IOL 297) were performed to aid chlamydial evaluation but the results are not presented here.

A further endocervical swab was taken for tissue culture of herpes simplex virus. High vaginal swabs were taken and inoculated on to Sabouraud's medium and into trichomonas medium ( $\mathrm{Lab} \mathrm{M}$ ) for culture of $C$ albicans and $T$ vaginalis respectively. After taking the swabs and the smear a full colposcopic assessment of the cervix was performed.

\section{ATTENDERS IN GENERAL PRACTICE}

One hundred and ninety seven asymptomatic patients attending for routine cervical smears were enrolled over two years in a single urban general practice in north west Glasgow comprising three partners and one trainee and with a list size of 5500 . Patients with abnormal cervical smears were routinely referred to the colposcopy department at Stobhill Hospital. The enrolled patients with normal cytology were matched for age with those enrolled at the colposcopy clinic (range 19-58 years; mean 30 ). All socioeconomic classes were represented, most patients being in social class III. Endocervical and high vaginal swabs were taken and placed in Stuart's transport medium for culture of $N$ gonorrhoeae, $C$ albicans, and $T$ vaginalis. Swabs taken from the endocervix were placed in chlamydial transport medium for culture of chlamydiae. Both Stuart's medium and chlamydial swabs were delivered to the laboratory the same day. Blood samples taken for serological study were analysed for chlamydial antibodies as described above. A Papanicolaou smear was taken with an Ayres spatula.

\section{MANAGEMENT OF INFECTION}

In both groups patients diagnosed as having $N$ gonorrhoeae were referred to the genitourinary physicians. Patients with $C$ trachomatis and $T$ vaginalis were treated appropriately, as were their partners. Those patients in whom infection with $C$ albicans was giving rise to symptoms were treated whereas patients in whom the infection was not causing symptoms were not.

\section{STATISTICAL ANALYSIS AND ETHICS}

Statistical analysis was by Fisher's exact test or the $\chi^{2}$ test, as appropriate, depending on the number of positive results. p Values $\leqslant 0.05$ were taken as significant.

The study was approved by the ethical committee, and all patients gave informed consent before enrolment.

\section{Results}

The table gives the culture and serological results in the two groups of patients. There was no significant difference between the groups in the results for $C$

Culture and serological results in the two groups of patients

\begin{tabular}{lcc}
\hline & $\begin{array}{c}\text { No (\%) of women } \\
\text { attending } \\
\text { colposcopy clinic } \\
(\mathbf{n}=101)\end{array}$ & $\begin{array}{c}\text { No (\%) of women } \\
\text { attending general } \\
\text { practitioner } \\
(\mathbf{n}=197)\end{array}$ \\
\hline C trachomatis (culture) & $6(6)$ & $24(12)$ \\
C trachomatis IgM positive & $1(1)$ & $8(4)$ \\
C trachomatis IgG titre $>128$ & $12(12)$ & $23(12)$ \\
C trachomatis IgG titre 16-128 & $19(19)$ & $36(18)$ \\
C trachomatis IgG titre $<16$ & $70(69)$ & $138(70)$ \\
Traginalis (culture) & $1(1)$ & $6(3)$ \\
N gonorrhoeae (culture) & $9(9)$ & $2(1)$ \\
C albicans (culture) & & $33(17)$ \\
Herpes simplex virus (culture) & & Not done \\
\hline
\end{tabular}

There was no significant difference between the groups in the prevalence of any infection studied. trachomatis. Culture and serological results are not mutually exclusive whereas serological findings are. $C$ trachomatis was cultured in six of the 101 patients with abnormal cervical smears and $24(12 \%)$ of the 197 patients with normal smears. Thirteen patients with abnormal smears and $31(16 \%)$ with normal smears had serological findings suggestive of probable current or recent infection (IgM positive or IgG titre $>128$ ). On summing all serological tests antibodies to $C$ trachomatis were detected in 32 patients $(32 \%)$ with abnormal smears and 67 patients (34\%) with normal smears.

There was also no significant difference between the groups in the prevalences of infection with $N$ gonorrhoeae, $T$ vaginalis, and $C$ albicans (table). The findings, however, disclosed an appreciable silent problem within general practice.

\section{Discussion}

This study found no significant difference in infection rates between patients with normal and abnormal cervical smears. A high prevalence of genital $C$ trachomatis infection $(6 \%$ and $12 \%$ of patients in the two groups respectively) was determined by culture in asymptomatic patients in the Glasgow area.

The serological test used was the microimmunofluorescence test (Micro-IF) developed by Wang and Grayston, ${ }^{7}$ which has become established as a sensitive and specific test for chlamydial antibodies. Although useful in diagnosing recently acquired chlamydial infection in men with urethritis, ${ }^{8}$ the diagnostic use of this test in women is impaired by high background rates of chlamydial antibodies which can be measured in the absence of chlamydial isolation. ${ }^{9}$ The lack of specificity seen in some antibody studies may be explained by persistent antibody responses with or without chronic infections of internal organs, previously treated infections, and serological cross reactions with other non-genital chlamydial strains. Using "single antigen" or "whole inclusion" serological tests is likely to underestimate or overestimate respectively the prevalence of chlamydial antibodies specific for type. The microimmunofluorescence test with multiple antigens is a more reliable guide to type specific antibodies and has been particularly useful in prevalence studies such as this and in diagnosing chlamydial pneumonitis in neonates and the FitzHugh-Curtis syndrome (perihepatitis) in women. The interpretation of chlamydial serological results, however, remains contentious.

In this study and with our criteria for interpreting the results of serological tests for $C$ trachomatis, the prevalence of recent infection was increased by a further $4-6 \%$. There also seemed to be a high rate of past infection (31 cases $(31 \%)$ in the group with abnormal cervical smears, 59 cases $(30 \%)$ in the group with normal smears). The high prevalence of chlamydial infection in our study, in other studies in the United Kingdom, and in other Western countries may warrant routine screening in women attending primary health care clinics. Although variations are seen due to differing demographic variables, factors associated with an increased risk of chlamydial infection are age (24 years or less), single marital state, use of oral contraceptives or no contraception, low socioeconomic class, intercourse with a new partner in the preceding two months, and living in an urban area. ${ }^{10}$ Some studies have found positive predictive variables for chlamydial infection ${ }^{11}$ whereas others have not. ${ }^{12}$ The decision whether to screen asymptomatic women should be based on the local prevalence rate. The high prevalence of chlamydial infection found in our series was presumably due to the population studied - that is, inner city, predominantly social class III. 
Chlamydial infection is an important source of gynaecological morbidity, being commonly associated with acute and chronic pelvic inflammatory disease and therefore infertility. ${ }^{13}$ Both problems have a large impact on the provision of gynaecological services in a given area. Screening and treatment for chlamydial infection before transcervical instrumentation that breaches the internal cervical os may prevent iatrogenic pelvic inflammatory disease, ${ }^{14}$ especially when retained products of conception within the uterine cavity provide an ideal medium for bacterial growth. We think that in cases of termination of pregnancy our results justify prior screening for chlamydia in the west of Scotland and in other areas with similar prevalence rates. If screening facilities are lacking, prophylaxis may be justified. Screening has been endorsed by workers elsewhere in the United Kingdom. ${ }^{3}$

The high percentage of women with asymptomatic genital chlamydial infection makes the use of effective antibiotics-for example, a tetracycline or erythromycin-important in cases of pelvic inflammatory disease and suspected postpartum and postabortion endometritis. Treatment of the partners of patients with genital chlamydial infection is recommended in order to prevent reinfection and reduce the pool of genital chlamydial strains within the general population. For the gynaecologist this may entail shared management with the genitourinary physicians. In our study the prevalence of other sexually transmitted diseases was very low, which may reflect regional differences. This low prevalence, however, should not be regarded as unimportant.

We have detected a high incidence of chlamydial infection in both general practice and colposcopy clinics in Glasgow. General practitioners and gynae- cologists should be more aware of the importance of investigating and treating chlamydial infection in both asymptomatic women and their partners.

We thank Dr I Tait, of Glasgow Royal Infirmary, for advice and the staff of the colposcopy clinic and laboratories for their help.

1 Longhurst HJ, Flower $\mathrm{N}$, Thomas BJ, et al. A simple method for the detection of Chlamydia trachomatis infections in general practice. $\exists R$ Coll Gen Pract of Chlamydia trach

2 Krogh C, Van Kirk J. A study of routine gonorrhea cultures in a family practice. 7 Fam Pract 1987;24:597-600

3 Fish ANJ, Fairweather DVI, Oriel JD, Ridgway GL. Chlamydia trachomatis infection in a gynaecology clinic population: identification of high-risk groups and the value of contact tracing. European fournal of Obstetrics and Gynaecology 1989;31:67-74.

4 Ridgway GL, Mumtaz G, Stephens RA, Oriel JD. Therapeutic abortion and chlamydial infection. BMF 1983;286:1478-9.

Mills A. Therapeutic abortion and chlamydial infection. BMF 1983;286:1649.

6 Alberico S, Facca MC, Di Bonito L, Millo R, Casaccia R, Mandruzzato GP. Frequency of cervicovaginal infections in cervical intraepithelial neoplasia. Eur J Gynaecol Oncol 1988;9:252-7.

7 Wang S-P, Grayston T. Human serology in Chlamydia trachomatis infection with microimmunofluorescence. $\mathcal{F}$ Infect Dis 1974;130:388-97.

8 Bowie WR, Wang S-P, Floyd AJ, et al. Etiology of non-gonococcal urethritis: evidence for Chlamydia trachomatis and Ureaplasma urealyticum. $\mathcal{F}$ Clin Invest 1977;59:735-42.

9 Schacter J, Cles L, Ray R, Hines PA. Failure of serology in diagnosing chlamydial infection of the female genital tract. $\mathcal{F}$ Clin Microbiol 1979;10: $647-9$.

10 Aral SO, Holmes KK. Epidemiology of sexually transmitted diseases. In: Holmes KK, Mardh PA, Sparling PF, Weissner PJ, eds. Sexually Holmes KK, Mardh PA, Sparling PF, Weissner PJ,
transmitted diseases. New York: McGraw-Hill, 1984:126-41.

11 Handsfield HH, Jarman LL, Roberts PL, et al. Criteria for selective screening for Chlamydia trachomatis infection in women attending family planning clinics. JAMA 1986;255:1730-4.

12 Wood PL, Hobson D, Rees E. Genital infections with Chlamydia trachomatis in women attending an antenatal clinic. Br $\mathcal{F}$ Obstet Gynaecol 1984;

13 Hare MJ. Pelvic inflammatory disease. BMF 1986;293:1225-8.

14 Southgate L, Treharne J, Williams R. Detection, treatment, and follow up of women with Chlamydia trachomatis infection seeking abortion in inner city general practices. BMF 1989;299:1136-7.

(Accepted l November 1990)

\title{
Ventilatory function as a predictor of fatal stroke
}

\author{
David P Strachan
}

Abstract

Objective-To investigate the relation between ventilatory function and subsequent mortality due to cerebrovascular disease.

Design-Prospective longitudinal study.

Subjects-A total of 18403 male civil servants aged 40-64 years at entry examination for the Whitehall study.

Main outcome measure-Mortality from cerebrovascular disease (ICD8 430-438) after 18 years of follow up.

Results-In all, 262 men with sinus rhythm at entry died due to stroke during the $\mathbf{1 8}$ years of follow up. Compared with men with a forced expiratory volume in one second of $\geqslant 3.5$ litres those with a value of $<3.0$ litres were almost twice as likely to die of cerebrovascular disease (rate ratio adjusted for age and systolic blood pressure $=1.88,95 \%$ confidence interval 1.32 to 2.69 ). This increased risk occurred within each tertile of systolic blood pressure. Nested case-control analyses were used to control precisely for confounding effects of age, height, and smoking (by matching) and employment grade and physiological risk factors (by modelling). The effect of forced expiratory volume in one second was independent of age, height, smoking habits, employment grade, blood pressure, weight, cholesterol concentration, glucose tolerance, electrocardiographic abnormalities, history of chest pain, and history of intermittent claudication.
Conclusions-Measurements of ventilatory function may assist clinical decisions about whether to treat mild hypertension. Impaired ventilatory function and stroke may share common causes.

\section{Introduction}

Reduced ventilatory function is a predictor of mortality due to cardiovascular causes and non-fatal coronary heart disease. ${ }^{1-8}$ Four studies have reported specifically the relation of ventilatory function to subsequent fatal ${ }^{6}$ or non-fatal ${ }^{259}$ cerebrovascular disease. An inverse association between spirometric indices and stroke was found in each case, although in none did it reach conventional levels of significance when adjusted for other risk factors.

This paper explores the relation between ventilatory function and mortality due to cerebrovascular disease among a cohort of middle aged civil servants who were followed up for 18 years. It also updates earlier findings from this cohort that related cardiovascular risk factors to fatal stroke. ${ }^{10}$

\section{Methods \\ STUDY POPULATION}

For the Whitehall study death certificates were obtained for 19018 male civil servants who were examined during 1967-9. ${ }^{.1}$ This analysis was limited to the 18403 men aged 40-64 years at examination and to deaths 